\title{
The Use of Negative Stain Electron Microscopy for the Examination of Pharmaceutical Preparations
}

R.C. Hamilton and M.B. Walker

Research and Development, CSL Limited, 45 Poplar Road, Parkville, Victoria. 3052, Australia

Negative stain electron microscopy is a quick and simple technique suitable for examining pharmaceutical preparations such as vaccines. It is especially appropriate when those preparations contain, viruses, subviral particles, virus-like particles, and particles of a size similar to viruses. A variety of chemicals can be used as negative stains. Routinely we use sodium phosphotungstate as a negative stain to examine preparations of influenza virions and sub-viral particles, and ammonium molybdate for preparations containing other virions.

ISCOMATRIX ${ }^{\mathrm{TM}}$ adjuvant consists of saponin, cholesterol and phospholipid, which under defined conditions form cage-like structures typically $40 \mathrm{~nm}$ in diameter. We use sodium phosphotungstate at pH 7.0 to stain samples of ISCOMATRIX ${ }^{\mathrm{TM}}$ adjuvant (Fig. 1). A variety of antigens can be formulated with ISCOMATRIX ${ }^{\mathrm{TM}}$ adjuvant to produce ISCOMATRIX ${ }^{\mathrm{TM}}$ vaccines[1]. Various ISCOMATRIX ${ }^{\mathrm{TM}}$ vaccines have been tested in animal models and in human clinical trials. Some antigens are stable at acid $\mathrm{pH}$ while other antigens are only stable at alkaline $\mathrm{pH}$. Cage-like structures can be observed in these vaccines using sodium phosphotungstate at $\mathrm{pH}$ 7.0. We investigated whether the cage-like structures exsisted in vaccines at the $\mathrm{pH}$ at which the vaccines were formulated. ISCOMATRIX ${ }^{\mathrm{TM}}$ vaccines in acid $\mathrm{pH}$ were stained with uranyl acetate and uranyl formate, and ISCOMATRIX ${ }^{\mathrm{TM}}$ vaccines in alkaline $\mathrm{pH}$ were stained with sodium tetraborate. Cage-like structures were observed in the ISCOMATRIX ${ }^{\mathrm{TM}}$ vaccine formulated at acid $\mathrm{pH}$ and stained with uranyl acetate (Fig. 2) and also in an ISCOMATRIX ${ }^{\mathrm{TM}}$ vaccine formulated at alkaline $\mathrm{pH}$ and stained with sodium tetraborate (Fig. 3).

Apo A-1 is the major protein constituent of the antiatherogenic HDL and is a primary candidate for the development of pharmaceuticals for the treatment of cardiovascular diseases [2]. Plasma HDL are spherical particles with a diameter of 7-13 nm. These spherical particles are derived from discoidal "nascent" HDL. To visualise discoidal complexes in rHDL sodium phosphotungstate is used as a negative stain [3] (Fig. 4). Harris [4] recommends a combination of cobalt nitrate and ammonium molybdate as an interesting negative stain for the study of liposomal suspensions. We evaluated this stain with ISCOMATRIX ${ }^{\mathrm{TM}}$ adjuvant and rHDL. Using this stain the discoidal complexes of rHDL were not observed but more complex structures were apparent (Fig. 5). Typical cage-like structures were observed with ISCOMATRIX ${ }^{\mathrm{TM}}$ adjuvant (Fig. 6).

Negative stain electron microscopy is a quick and simple technique for examining pharmaceutical preparations. Some samples show a consistent morphology no matter what stain is used. In other samples the morphology observed depends on the negative stain that is used.

\section{References}

[1] M.J. Pearse and D. Drane, Advanced Drug Delivery Reviews 57 (2005) 465

[2] C.R. Sirtori et al. , Atherosclerosis 142 (1999) 29

[3] A.V. Nichols et al. , Biochim. Biophys Acta 750 (1983) 353

[4] J.R. Harris Negative Staining and Cryoelectron Microscopy, Bios Scientific Publishers Limited, Oxford, 1997 


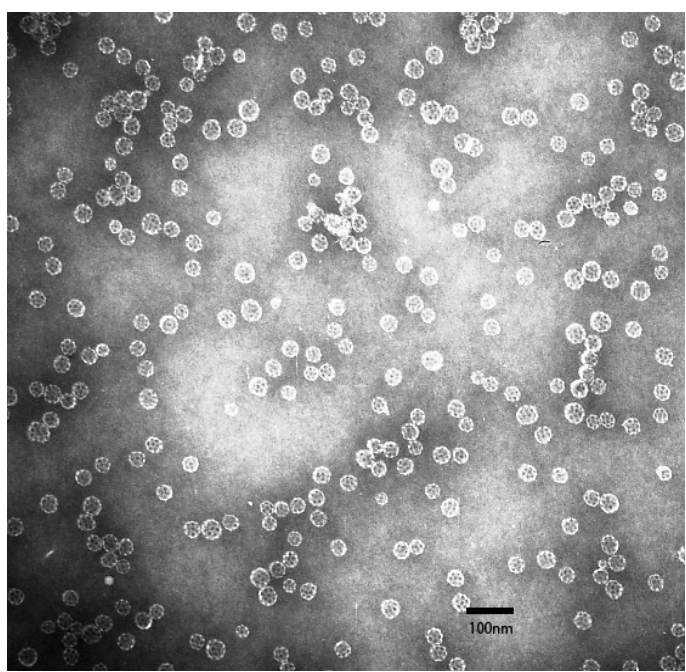

FIG 1: ISCOMATRIX ${ }^{\mathrm{TM}}$ adjuvant stained with sodium phosphotungstate at $\mathrm{pH} 7.0$

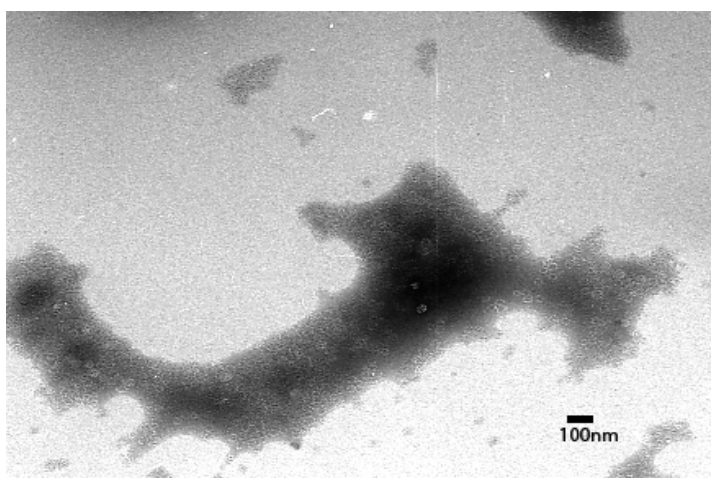

FIG 3: ISCOMATRIX ${ }^{\mathrm{TM}}$ vaccine formulated at alkaline $\mathrm{pH}$ and stained with sodium tetraborate

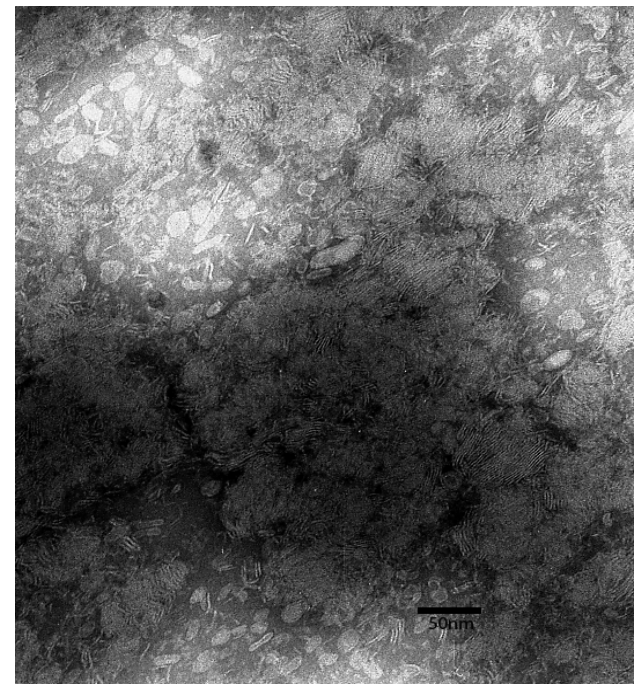

FIG 5: rHDL stained with a combination of cobalt nitrate \& ammonium molybdate
927605500114 Published online by Cambridge University Press

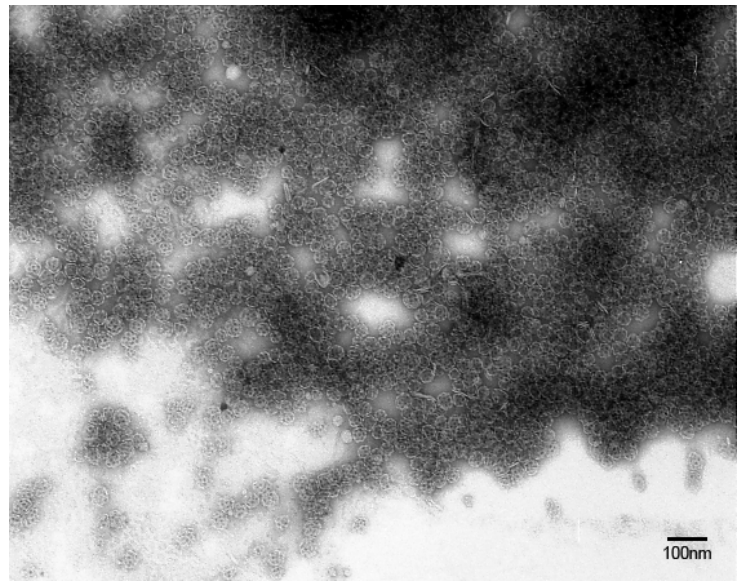

FIG 2: ISCOMATRIX ${ }^{\mathrm{TM}}$ vaccine formulated at acid $\mathrm{pH}$ and stained with uranyl acetate

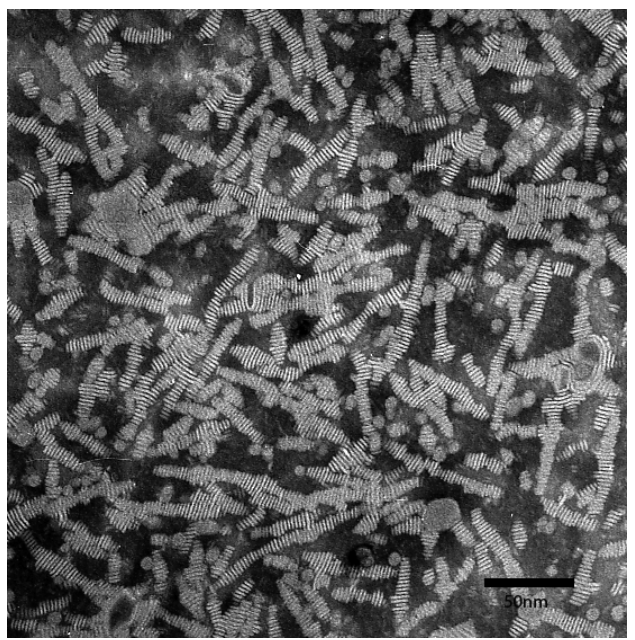

FIG 4: rHDL stained with sodium phosphotungstate, $\mathrm{pH} 7.0$

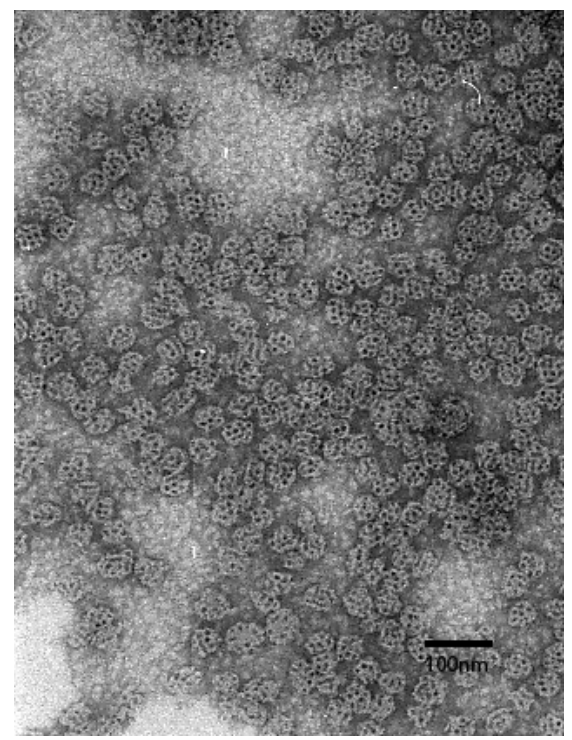

FIG 6: ISCOMATRIX ${ }^{\mathrm{TM}}$ adjuvant stained with a combination of cobalt nitrate and ammonium molybdate. 